\title{
Using ultrasound to image the foot in rheumatoid arthritis: current understanding, challenges and future scope
}

Understanding of the use of diagnostic ultrasound (US) in rheumatology has grown significantly in recent years. As a result, there has been an increase in focus on the use of US techniques in the assessment and diagnosis of foot disease associated with rheumatoid arthritis (RA). There is great potential for the use of diagnostic US in aiding current assessment of RA foot disease with the ultimate outcome of more effective management of foot and ankle symptoms. In this article, the authors discuss the use of US as a key modality in the investigation of RA foot disease and highlight how advancing techniques in the use of US, which are evolving from general rheumatological practice, may be applied to RA foot disease.

\section{KEYWORDS: foot foot disease rheumatoid arthritis synovitis ultrasound}

The past decade has seen significant advances in ultrasound (US) imaging for the evaluation of joint damage and inflammation in rheumatoid arthritis (RA). Using US, the diagnosis of joint disease and the assessment of joint damage and inflammation in patients with RA has been transformed [1-3]. Recently, some excellent narrative reviews have been published related to the use of US in rheumatology $[1,2,4,5]$; however, none are specific to US assessment of the foot in RA. Several authors have described the use of US to identify soft tissue pathology within the foot [4-7] and there is an emerging body of evidence that advocates the use of US investigation of the foot in RA. The purpose of this review is, therefore, to examine the current emerging evidence related to US investigation of the foot in RA and to consider a future scope for this.

\section{Rheumatoid arthritis}

RA is the most common cause of inflammatory arthritis with a prevalence of approximately $1 \%$ of adults [8]. The disease typically involves small joints of the hands and feet. In the UK, there are approximately 12,000 new cases diagnosed annually, with women affected more commonly than men [9]. Inflammation of the synovial membrane of joints (synovitis) with erosion of adjacent bone is the hallmark of the disease [10]. The precise detection of synovitis has become fundamental to the management of RA and work continues to develop US techniques to do this $[1,3]$.

\section{Summary of US use in rheumatoid arthritis}

Grayscale (GS) and power Doppler (PD) US are established imaging techniques in routine clinical practice for rheumatologists $[1,2,5]$. GS (brightness mode [B-mode]), refers to images that are produced in a black and white format; each white dot in the image represents a reflected sound wave [1]. PD measures the amplitude of the Doppler signal (determined by the volume of blood flow) and this is superimposed on the GS image, thereby depicting microvascular blood flow [1]. It has been suggested that US has advantages over MRI owing to its real-time imaging capabilities, chair-side accessibility, reduced scanning time, low acquisition cost and ability to simultaneously scan bone and soft tissues in GS or PD with enhanced inflammatory feedback [11-14]. Comparative sensitivity, specificity and accuracy of US versus MRI range from 80-97\% across various anatomical pathological details to 60-98\%, respectively, suggesting comparable clinical and research usage [11-15].

The use of US to detect pathology in RA that is not clinically apparent and to guide treatment decisions has gained recognition [16]. The impact of this on rheumatological practice can be clearly seen in the shift towards the inclusion of US assessment in National and International guidelines, the most notable being the criteria for diagnosis [17]. Previously, criteria for diagnosis of early RA involved the demonstration of bone erosion shown radiologically by x-ray [18]. However, as imaging techniques evolved, the criteria were criticized for their reliance on conventional radiography, which is insensitive for the detection of synovitis [19-21]. In 2010, new classification criteria were devised in which reference to use of US "for confirmation of the clinical findings" was made [17]. Following this, in a 1-year prospective study of 109 RA patients,

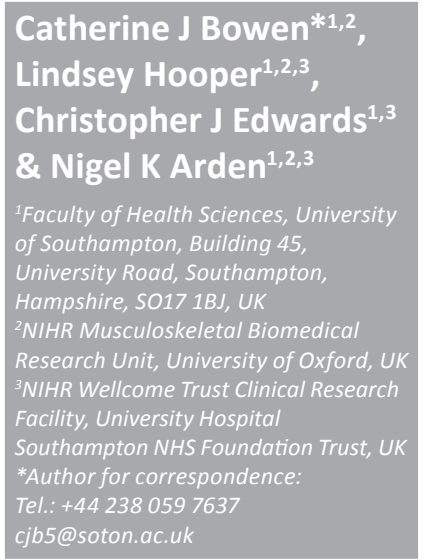

Future $\because:$ f 
investigators demonstrated that US assessment may actually improve the diagnostic accuracy of the criteria [22].

Advances in PD US imaging have enabled better assessment of changes in joints and soft tissues due to inflammation such as effusions, proliferating synovium and active synovitis $[23,24]$. Using high-end scanners, PD US is reportedly comparable to MRI scores in monitoring changes in RA disease state [25]. Current strategies for the management of RA require early, accurate detection and quantification of inflammation $[1,2,16]$. The detection of changes in synovial perfusion by PD US techniques, particularly in early disease, is agreed to be advantageous in informing interventions for 'tight control' of disease activity [24,26]. Other researchers have also demonstrated that the presence of PD US activity is the most accurate determinant of flare in RA patients otherwise suspected to be in remission [27]. Conversely, as targeted treatment is encouraged, investigators and clinicians utilizing the technique also warn that interpretation of PD US can be challenging owing to frequent presence of artifact [24].

Despite the increasing evidence supporting the use of US in the evaluation of RA, there remains a lack of standardization between studies [7]. For example, on GS US, synovitis may be detected by when it is thickened (synovial hypertrophy) as it appears as hypoechoic intra-articular tissue $[1,2]$ such that detection of synovial hypertrophy by GS US, without PD, can be found in healthy subjects. Interestingly, within the foot, normal limits for metatarsophalangeal (MTP) joint synovial hypertrophy have been recorded by US as $2.9 \mathrm{~mm}$ or less in healthy subjects [28].

\section{Current evidence for use of US to detect foot pathology in RA}

It is evident that US is routinely used to measure the anatomical extent of inflammation and damage in early inflammatory arthritis, monitor the course of joint disease and determine the efficacy of drug therapies. The prevalence of foot pathology and foot symptoms in RA is well documented in the literature and it is generally agreed that the majority of patients are ultimately affected, although reported figures on the prevalence of foot involvement in RA is varied. In a recent survey of 395 patients with RA in the UK, almost $94 \%$ of patients had experienced foot symptoms during the course of the disease and $35 \%$ had reported foot pain as the presenting symptom of RA [29]. The manifestations of RA may include pain, joint deformity, callus formation and ulceration [30-32], which may lead to impairment of gait [33,34] and other interruptions in daily activities of living, such as loss of independence [29,35]. US evidence suggests that synovitis is most frequently detected in the second MTP joint and that the fifth MTP joint is the most common site of erosion [36,37]. Siddle et al. confirmed this using 3T MRI, demonstrating that the fifth MTP joint was the most common site for forefoot damage in RA [38].

Work continues to be applied to investigate US detectable pathology within the foot that is otherwise unseen by clinicians [11,39-41]. The prevalence of foot involvement and the extent of foot symptoms in RA patients is notably underestimated by clinicians [29] and it is widely accepted that, in patients with RA, clinical examination and conventional radiography may be relatively insensitive for the detection of joint effusion and synovitis $[16,42]$. For example, US has been reported to be ten-times more likely to detect MTP joint synovitis [43] and five-times more likely to detect thickening of forefoot bursae synovium than clinical examination alone [44].

US is also useful in supporting the clinical diagnosis of RA foot disease. In a prospective controlled observational study, Matsos et al. reported a significant increase in confidence by rheumatologists using US to diagnose synovitis, tenosynovitis, erosions and enthesitis within the feet of 62 patients [45]. The inferences from these findings suggest good clinical utility for the use of US in assisting the diagnosis of foot pathology and ensuring appropriate foot structures are injected/treated. However, it should be noted that with only two study rheumatologists, the external validity of their findings may be limited [45].

Typically, US investigations of foot involvement in RA focus on characterizing the pattern of involvement of the forefoot; especially the MTP joints $[4,37,41,46]$. Plantar forefoot bursitis (anatomical intermetatarsal and adventitial plantar fat pad bursitis) related to RA has also recently received more attention $[41,47]$. Other common causes of symptoms in this area in adults that may not be directly attributable to the manifestations of RA within the foot, include Morton's neuroma, metatarsal stress fracture or predislocation syndrome (with associated capsulitis and disruption of the plantar plate) of the MTP joints [48-50]. Further soft tissue structures within the plantar forefoot area that may be detectable by US, but not directly related to the pathological manifestations of RA, are the dorsal and plantar interossei muscles, lumbrical muscles and the oblique head of the adductor hallucis muscle [51]. 
For the midfoot or rearfoot, in their systematic review of US assessment of synovitis, Joshua et al. reported that few data existed, although the review only focused on US assessment of synovitis for the hand, wrist, elbow, shoulder, knee, ankle and foot joints, and was conducted over 5 years ago [7]. Others have since argued the advantages for the use of US for imaging of the midfoot and rearfoot [52]. In patients with RA, US has been useful to image tenosynovitis, especially within the foot and ankle tendons [5,53-58]. In a small study of patients with established RA, US demonstrated good correlation with MRI for identifying hindfoot pathology [11]. Using US, Riente et al. investigated the foot joints in 100 patients with established RA [36]. Among the midfoot, the talonavicular joint was the more frequently involved, with US-detected synovitis affecting 38\% of observed cases. The investigators rarely observed effusion at the intermediate and lateral cuneiform-metatarsal joint and the cuboid-metatarsal 4 and 5 joints. Erosion was only in the lateral cuneiform-metatarsal joint and the cuboid-metatarsal 5 joints [36].

While evidence advocating the use of US imaging for RA foot disease is accumulating, most studies have tended to focus on the diagnostic accuracy of US in detecting specific foot pathologies in RA or reporting of techniques to detect pathology. Investigations in this field are mostly cross-sectional in design and, although there is concerted international effort to address these issues [3,16], relatively few data exist that demonstrate support for the use of US in the assessment, diagnosis and management of RA foot disease over time. However, as demonstrated by Hooper et al., US investigation of the forefoot has the potential to contribute to the assessment of prognosis [41]. In a longitudinal study of 149 patients, Hooper et al. demonstrated that the presence of US-detected foot pathology can significantly predict patient-reported foot related disability after 3 years [41]. This highlights the potential value in applying US to foot assessment, although in the absence of other longitudinal data, the relationship between USdetected foot disease, poor clinical symptoms and foot disability remains speculative.

\section{US characteristics of foot pathology in RA}

Riente et al. provides a detailed documentation of a proposed scanning protocol for the foot [6]. Additionally, the US imaging characteristics of the normal anatomical structures of the foot are well described [5] as well as techniques for imaging the small joints of the forefoot [4] and the ankle and foot [5]. Various internet learning resources also exist that give examples of US images of normal foot anatomy as well as images of musculoskeletal foot pathology [101-103].

Classical GS US appearances of joint structures demonstrate hyperechogenicity at the bony margins due to the increased refraction of sound waves off the dense cortical bone, with hypoechoic joint centers [1,2,59]. Such images demonstrate uniformity in acoustic feedback, where there is homogeneity across the cortical bone surface. Some authors report that foot structures are readily accessible to US examination as they are relatively superficial $[4,5,36]$. Comparative GS and PD appearances within the first MTP joint in a patient with RA are illustrated in Figure 1.

By contrast, others have reported that differentiation between closely aligned anatomical structures that may be disrupted with RA diseaserelated deformity can be challenging $[49,60,61]$. If deformity of the foot joints, such as retraction of the lesser digits, exists inaccurate transducer orientation complications, such as anisotropy (US beam strikes the anatomical structure at an angle of less than $90^{\circ}$ so that the sound waves are scattered rather than being reflected back to the transducer), acoustic shadowing (signal loss after the US beam has hit a highly reflective surface) or ghosting (refracted US beam giving image duplication or triplication) can occur $[6,39,49,62]$.

User techniques such as transducer nonperpendicular orientation or 'heel-toeing' (the rocking of the transducer to displace soft tissues) may be used beneficially to enhance tissue recognition [6]. It is also recommended that real-time dynamic imaging and tissue compression are used to improve US scanning specificity [6,63]. Tissues such as the plantar flexor digitorum brevis tendons (appearing as fibrillar structures with or without superficial acoustic shadowing) or fibrous tissue around the neurovascular bundles (a complex hypoechoic mass consistent with nonpathological nerve tissue) are particularly susceptible to user error, making definitive diagnoses challenging [6,49]. In these instances, the use of real-time dynamic imaging for the assessment of compressibility can improve diagnostic accuracy [41].

\section{US assessment of inflammation versus mechanical impairment within the foot}

Researchers utilizing US techniques suggest that, in patients with RA, a combination of both mechanical and inflammatory factors lead to the development of soft tissue 


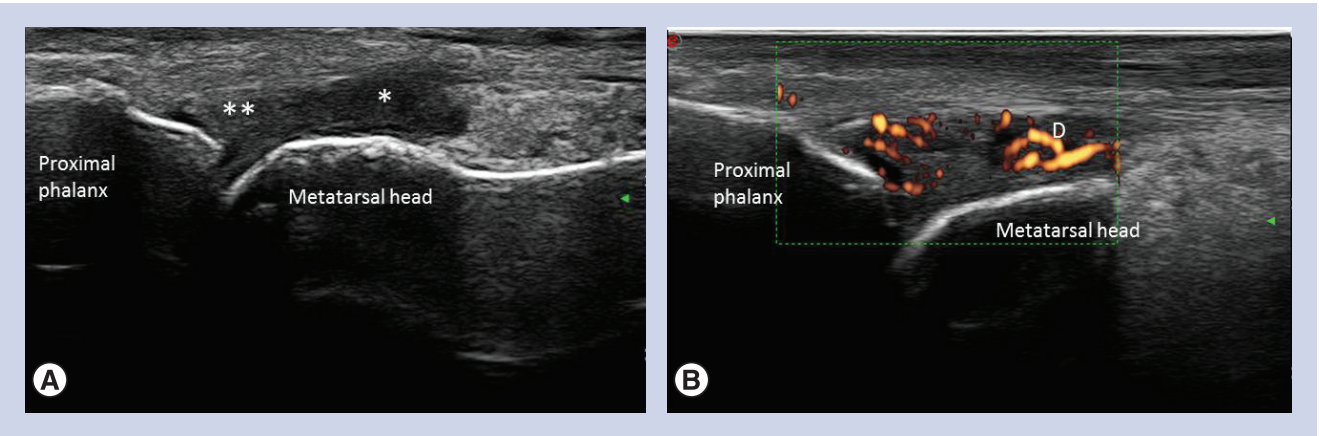

Figure 1. Grayscale and power Doppler ultrasound images of the first metatarsophalangeal joint in a patient with rheumatoid arthritis (dorsal longitudinal scan). (A) Grayscale ultrasonography demonstrates anechoic effusion $\left({ }^{*}\right)$ with hypoechoic synovial thickening $(* *)$.

(B) Increased vascularization of thickened synovium as evidenced by the presence of power Doppler signal (D).

pathology within the foot [41]. US investigators have hypothesized that MTP joint synovitis is initiated by the pathological processes that are active in early RA, but then perpetuated, independently of the disease, linked to underlying structural and functional mechanical irritation [39]. Imaging modalities are, therefore, not the only technologies that have contributed towards understanding the pathological processes occurring within the forefoot in RA. Instrumentation for gait mechanics $[33,34,64]$, foot pressure analysis [40] and patient facing questionnaires $[65,66]$ have proven to be invaluable in aiding and augmenting investigations of inflammation and mechanical impairment within the foot.

Recently, there have been increases in the reporting of novel combinations of US imaging and functional assessment to describe foot pathology. Interface foot pressures are readily measured within the clinical setting, especially with the development of wireless technology [67]. Often, patients with RA load their forefeet medially during walking, yet the most frequent sites of USdetected forefoot pathology are in the lateral forefoot segments [40]. In this patient group, clinical strategies to offload high foot pressures over time would benefit from additional information from US imaging to prevent overloading of soft tissue inflammation that may not be detected clinically.

The use of high-specification 3D kinematic motion capture technology has allowed more specific analysis of gait strategies adopted by patients who have RA and further exploration of the impact of RA on foot mechanics $[33,34,68,69]$. Subsequently, a small number of studies have used 3D motion analysis in combination with US [32,55] or MRI [70] to determine associations between foot pathology in RA and function. However, to date, this area of work is largely developmental and remains limited by ability of the technology to reliably assess the segmental motion of the midfoot and forefoot during gait.

\section{US use in assessing efficacy of interventions for RA foot disease}

A key paradigm shift in the management of foot pathology related to RA is in the advocacy for early detection, targeted therapy and tight control of foot disease, mimicking the medical model for exploiting the 'therapeutic window of opportunity' [71].

There is potential for the routine use of US to be an integral tool towards facilitating tight control of RA foot disease and the evaluation of management [52,71-74]. Emerging evidence suggests that the use of US provides clinicians with the diagnostic capabilities to detect, grade and monitor active RA foot disease to expedite timely interventions $[39,41,47,55]$. Unfortunately, while US imaging has contributed towards developing understanding of inflammatory and mechanical processes in RA foot disease, little evidence exists related to the use of US in determining response to nonpharmacological interventions, such as foot orthoses and footwear modifications.

More emphasis has been placed on using US as a useful adjunct to clinical examination in monitoring the course of RA disease and determining efficacy of pharmacological interventions such as disease-modifying antirheumatic drugs and biological therapies [2]. Biological therapies have revolutionized the treatment of RA as these agents specifically inhibit inflammatory cytokines, immune cell interaction or deplete immune cells, and are highly effective when conventional antirheumatic drugs have failed to control RA [75-77]. However, it is possible that some patients with RA with active foot disease may fall below the threshold of eligibility 
for biological therapies or be falsely classified as being in remission [78]. The usual criteria for assessment of RA disease remission is based upon measurement that excludes the feet [79]. In such cases, US assessment to determine the extent of foot disease could be deemed essential to improve access to these drugs. Indeed, from initial data, investigators have shown that in a small sample of patients with established RA $(\mathrm{n}=31)$, MTP joint synovitis does respond to biologic therapies albeit at a much slower rate than indicated by markers of disease state [39].

An area where US use has expanded considerably as a useful adjunct in therapy for RA foot disease is in its utility for guiding injection techniques. Over the past decade, corticosteroid injections, joint aspiration and synovial biopsy have been reported as more readily performed on the foot under US guidance than by clinical palpation alone $[1,2,6,80,81]$. Investigators also agree that US guiding improves the efficacy of local corticosteroid injections, as well as improving diagnostic capability for inflammatory arthritis within the foot $[6,80,81]$. In patients with midfoot degenerative changes $(n=59)$, US observations frequently led to changes in diagnosis of inflammatory lesions in painful feet, as well as planning of corticosteroid injections [81]. Additionally, it has been proposed that the use of US in the management of corticosteroid injections in chronic inflammatory diseases may have an important prognostic and diagnostic impact on the efficacy of local treatment of painful foot joints [80].

As well as clinical applications, researchers have used US to guide injection of contrast agent into lesser MTP joints to enhance T1-weighted fat-suppressed sequences for investigation by MRI [70]. Nevertheless, despite such investigations and considering that appropriate therapy might strongly depend on accurate diagnosis, studies addressing the impact of US guidance for injection techniques in RA foot disease are rare and more data are required in this area.

\section{Challenges to the uptake of US as a key modality in imaging foot disease}

US-detected disease activity is emerging as a key outcome measure in determining and evaluating clinical management of RA [3,16,82]. It has been proposed that US evaluation of synovitis as an outcome measure has the potential to be as relevant as physical examination [82]. As with any emergent field, from the above discussion, it is evident that the efficacy of using diagnostic US to perform this is challenging, particularly in reference to foot pathology. The main concerns for the use of US in RA foot disease revolve around the need for agreement and uptake of clinically useful reference standards for US examination of feet.

The first challenge related to this is that the clinical utility of US is compounded in that it is highly operator dependent and there is a lengthy period required to develop the necessary skills $[2,83]$. As US technology has improved, clinical expertise in performing musculoskeletal US has advanced dramatically. This has reinforced the need for adequate training by rheumatologists to learn the techniques in order to improve their clinical practice [3]. As such, models to support the learning needs of rheumatologists in the use of US have been devised in Europe and the USA $[3,84,85]$. Additionally, the uptake of US by other nonmedical clinicians, such as rheumatology specialist nurses, physiotherapists and podiatrists, is gaining recognition [2]. A recent proposed framework for the development of competencies in US scanning techniques recognized that the single biggest obstacle to the uptake of US is the length of time taken to develop the necessary skills [86]. The authors recommended that learning in rheumatology could be tailored to areas directly relevant to a clinician's discrete field of practice [86]. The use of US assessment of the foot in clinical practice by clinicians who specialize in the foot, such as podiatrists, could therefore be beneficial to patients with RA in facilitating more effective timely referral, management and review of foot problems [62]. Clinicians such as podiatrists arguably have a discrete detailed anatomical knowledge of the foot and one study has demonstrated good reliability of a podiatrist tested against a radiologist $(\kappa: 0.702$; $\mathrm{p}<0.01)$ in the use of US for the evaluation of foot disease in RA [62].

A second challenge is that the uptake of US as an outcome measure is marred by a persistent lack of data regarding the metric properties of US in the evaluation of RA foot disease. Due to this, even the most recent recommendations for the use of US in the clinical management of RA state that conventional radiography should be used as the initial imaging technique to detect damage in the feet, and that US should be used secondary to conventional radiographic findings [16]. The Outcome Measures in RA Clinical Trials US Special Interest Group continues to focus its work on defining and standardizing approaches for US assessment [12,59,87]. Of interest is the investigation of extended US joint and tendon counts as a tool to assess response to therapy [88]. There is potential for the development of systematic US joint examinations to be 
adapted and applied to the foot. New US scoring systems that have been developed include a 44-joint count that comprises the MTP joints [89], a 38-joint count that comprises the MTP joints and ankle [90] and a 78-joint count that comprises the ankle (talocrural joint; anterior), four major foot joints (talonavicular, calcaneocuboidal, cuneonavicular and subtalar; anterior and lateral), tarsometatarsal 1-5 (dorsal), MTP joints 1-5 (dorsal) and the interphalangeal (dorsal) joint of the first toe [91]. Each score differs in terms of joints, tendons and bursae included and the different investigators have attempted to determine the optimal combination of joints and tendons to be assessed by US. To scan all structures in the 78-joint count would be extremely time consuming, especially when assessment of as few as seven joints and five tendons/tendon compartments is sensitive to changes in disease state [88]. A variety of reduced joint counts for scoring synovitis have emerged that correlate well with the larger more comprehensive joint counts [92-96], although they rarely include foot joints. The German US7 score, however, examines seven joints, two of which are within the foot (wrist metacarpophalangeal (MCP)2, MCP3, proximal inter-phalangeal (PIP)2, PIP3, MTP2 and MTP5) [94] and more recently US assessment of the ankle and second through the fifth MTP joints has been included in a reduced joint count [93]. Ideally, evaluation of the use of all the foot components of the 78-joint US count as a discrete score could be further explored. A validated discrete US foot joint count could be a valuable utility in moving the field forward towards early detection, targeted therapy and tight control of RA foot disease.

\section{Conclusion}

There is great potential for the use of US imaging by informed clinicians to aid the assessment and evaluation of RA foot disease. The ultimate aim being effective management of foot and ankle symptoms. US is a clinically accessible, real-time and relatively low-cost technique that can be used for the examination of RA foot disease. It is evident that most research to date has focused on the use of US toward further understanding RA foot disease, but many evidence gaps exist and more effort is required to develop these techniques for clinical utility over time. If these evidence gaps are addressed, US could emerge as a key modality revolutionizing the shift in management towards early detection, targeted therapy and tight control of RA foot disease. The technical advances expected in the future are likely to allow faster, more accurate imaging of foot joints and related structures, as well as differentiation of inflammatory and/or mechanical causes for symptomatic feet. The impact that could be achieved would be in improving mobility and health-related quality of life for patients with RA.

\section{Future perspective}

As US is fast becoming an essential tool in routine rheumatological clinical practice, scope exists for the further development of US to aid specific diagnosis of pathological sites and facilitate interventions that are targeted more precisely at the presenting problem within the foot. Within rheumatological practice, new developments include contrast-enhanced US, 3D and 4D US, elastography and fusion imaging $[2,3]$. It stands to reason that over the next 5-10 years US imaging of RA foot disease would follow this path too.

In rheumatology, application of contrastenhanced GS US is developing to maximize spatial resolution using the concept of 'microbubbles' $[2,3]$. The coupling of very high frequency transducers with US sensitive contrast agents increases the sensitivity of PD US feedback for the imaging of synovial membrane perfusion [97]. Thus using this technique it is possible to differentiate active synovitis from inactive intra-articular thickening [97]. A key issue in determining the status of RA foot disease is whether synovitis within the foot joints is caused by mechanical or inflammatory factors or a combination of both [41]. Contrastenhanced GS US is perhaps the most promising new technique that may decipher this problem, allowing treatments for RA foot disease to be appropriately and more precisely targeted at the underlying cause.

$3 \mathrm{D}$ and 4D US are also promising technologies that allow the visualization of cubes of echoes that can be explored on any plane to quantify regions of interest [98]. This would be of particular interest for the determination of the extent of foot pathology such as bursitis within the forefoot, as well as MTP joint synovitis [41,47]. For example, in patients with RA, within the forefoot it is hypothesized that different types of bursae exist, anatomical intermetatarsal and adventitial, and that both types cause symptoms, but may have different pathological causes and different mechanical properties [41].

The use of real-time elastography has not been explored within the foot; however, it may be a useful adjunct in differentiating pathological structures such as neuroma, rheumatoid nodules and bursitis in RA foot disease. Using elastography, analysis of US echo signals could be 
performed while the probe compresses or relaxes the tissue allowing in vivo assessment of the tissue's mechanical properties [13]. Fusion imaging is also a novel technique that may be useful for the further differentiation of pathological structures in RA foot disease, as has been demonstrated in visualization of hand and wrist bony deformities [99]. Fusion images are obtained by simultaneous mapping of US images onto a preacquired MRI or CT volume data set, thereby increasing the anatomical detail of US [3].

As technology continues to evolve, for the investigation of RA foot disease, the greatest potential lies in the fusion of US imaging techniques with functional assessment techniques. The mapping of real-time US-detected foot pathology with motion capture and in-shoe foot pressure technology to determine relationships between mechanical function and RA foot disease, has the capacity to revolutionize current clinical decision-making and treatment pathways for patients with RA. The aim is for a positive impact on patient mobility and activity participation.

\section{Disclaimer}

The content is solely the responsibility of the authors and does not necessarily represent the official views of National Institute of Health Research.

\section{Financial \& competing interests disclosure}

$L$ Hooper is currently supported by a National Institute of Health Research UK Clinical Doctoral Academic fellowship award. The authors have no other relevant affliations or financial involvement with any organization or entity with a financial interest in or financial conflict with the subject matter or materials discussed in the manuscript apart from those disclosed.

No writing assistance was utilized in the production of this manuscript.

\section{Executive summary}

\section{Current evidence for use of ultrasound to detect foot pathology in rheumatoid arthritis}

- Ultrasound (US) is an effective modality in characterizing specific foot pathology in rheumatoid arthritis (RA) where clinical assessment is relatively insensitive.

- Specific US scanning protocols and learning resources have been developed that are specific for the foot.

- US evaluation of the foot joints can yield beneficial information about RA disease state over and above that provided by investigation of other joints.

\section{US assessment of inflammation versus mechanical impairment within the foot}

- From evidence of US findings, there appears to be a complex interplay of inflammatory and mechanical factors that are associated with RA foot disease.

- Investigators are increasingly describing foot pathology using novel combinations of US techniques in conjunction with functional assessment and motion capture techniques.

\section{US use in assessing efficacy of interventions for RA foot disease}

- Emerging evidence suggests that the use of US provides clinicians with the diagnostic capabilities to monitor active RA foot disease following pharmacological intervention.

- Scope exists for the further development of US to monitor efficacy of nonpharmacological interventions such as foot orthoses and footwear adaptations.

- The use of US to guide injections has been proven to be highly effective for local treatment of painful foot joints.

Challenges to the uptake of US as a key modality in imaging foot disease

- The clinical utility of US by practicing clinicians is often challenged owing to the highly operator-dependent outcomes.

- Models to support learning needs for the uptake of US in discrete areas, such as the assessment of RA foot disease, are emerging, but require further validation.

- Validation of a discrete joint count for the assessment of RA foot disease is essential.

\section{Future perspective}

" New developments in US, such as the use of contrast-enhanced US, 3D and 4D US, elastography and fusion imaging, will be extremely beneficial in defining and characterizing different pathological structures in the foot.

- The greatest potential for investigation of RA foot disease lies in the merging of US imaging techniques with assessments of functional ability to differentiate inflammatory and noninflammatory RA foot disease.

\section{References}

Papers of special note have been highlighted as:

- of interest

" $"$ of considerable interest

1 Brown AK. Using ultrasonography to facilitate best practice in diagnosis and management of RA. Nat. Rev. Rheumatol. 5(12), 698-706 (2009).
2 Taggart A, Benson C, Kane D. Ultrasound in Rheumatology. Arthritis Research UK, Reports on the Rheumatic Diseases, Issue 9, Topical Reviews Series 6 (2011).

3 Kang T, Lanni S, Nam J, Emery P, Wakefield RJ. The evolution of ultrasound in rheumatology. Ther. Adv. Musculoskelet. Dis. 4(6), 399-411 (2012).
4 Mcnally EG. Ultrasound of the small joints of the hands and feet: current status. Skeletal Radiol. 37(2), 99-113 (2008).

5 Micu MC, Nestorova R, Petranova T et al. Ultrasound of the ankle and foot in rheumatology. Med. Ultrason. 14(1), 34-41 (2012). 
- Provides an excellent overview of scanning techniques for the foot, as well as indications for ultrasound (US) use in rheumatic diseases.

6 Riente L, Delle Sedie A, Iagnocco A et al. Ultrasound imaging for the rheumatologist $\mathrm{V}$. Ultrasonography of the ankle and foot. Clin. Exp. Rheumatol. 24(5), 493-498 (2006).

7 Joshua F, Lassere M, Bruyn GA et al. Summary findings of a systematic review of the ultrasound assessment of synovitis. J. Rheumatol. 34(4), 839-847 (2007).

- Includes a summary of the evidence for the use of US in detecting synovitis in some foot joints and provides clear recommendations for future investigations.

8 Gibofsky A. Overview of epidemiology, pathophysiology, and diagnosis of rheumatoid arthritis. Am. J. Manag. Care 18(Suppl. 13), S295-S302 (2012).

9 NICE. CG79 Rheumatoid Arthritis: The Management Of Rheumatoid Arthritis In Adults. NICE, London, UK (2009).

10 Tarner IH, Harle P, Muller-Ladner U, Gay RE, Gay S. The different stages of synovitis: acute vs chronic, early vs late and non-erosive vs erosive. Best Pract. Res. Clin. Rheumatol. 19(1), 19-35 (2005).

11 Wakefield RJ, Freeston JE, O'Connor P et al. The optimal assessment of the rheumatoid arthritis hindfoot: a comparative study of clinical examination, ultrasound and high field MRI. Ann. Rheum. Dis. 67(12), 1678-1682 (2008).

12 Wakefield RJ, D'agostino MA, Iagnocco A et al. The OMERACT Ultrasound Group: status of current activities and research directions. J. Rheumatol. 34(4), 848-851 (2007).

13 Cimmino MA, Grassi W. What is new in ultrasound and magnetic resonance imaging for musculoskeletal disorders? Best Pract. Res. Clin. Rheumatol. 22(6), 1141-1148 (2008).

14 Katz JD, Brindle K. Preface. MRI and ultrasound in diagnosis and management of rheumatological diseases. Ann. NY Acad. Sci. 1154, vii-viii (2009).

15 Szkudlarek M, Narvestad E, Klarlund M, Court-Payen M, Thomsen HS, Ostergaard M. Ultrasonography of the metatarsophalangeal joints in rheumatoid arthritis: comparison with magnetic resonance imaging, conventional radiography, and clinical examination. Arthritis Rheum. 50(7), 2103-2112 (2004).

16 Colebatch AN, Edwards C, Ostergaard M et al. EULAR recommendations for the use of imaging of the joints in the clinical management of rheumatoid arthritis. Ann. Rheum. Dis. 6, 1-11 (2013).
17 Aletaha D, Neogi T, Silman AJ et al. 2010 rheumatoid arthritis classification criteria: an American College of Rheumatology/European League Against Rheumatism collaborative initiative. Arthritis Rheum. 62(9), 2569-2581 (2010).

18 Arnett FC, Edworthy SM, Bloch DA et al. The American Rheumatism Association 1987 revised criteria for the classification of rheumatoid arthritis. Arthritis Rheum. 31(3), 315-324 (1988).

19 Backhaus M, Kamradt T, Sandrock D et al. Arthritis of the finger joints: a comprehensive approach comparing conventional radiography, scintigraphy, ultrasound, and contrast-enhanced magnetic resonance imaging. Arthritis Rheum. 42(6), 1232-1245 (1999).

20 Backhaus M, Burmester GR, Sandrock D et al. Prospective two year follow up study comparing novel and conventional imaging procedures in patients with arthritic finger joints. Ann. Rheum. Dis. 61(10), 895-904 (2002).

21 Wakefield RJ, Gibbon WW, Conaghan PG et al. The value of sonography in the detection of bone erosions in patients with rheumatoid arthritis: a comparison with conventional radiography. Arthritis Rheum. 43(12), 2762-2770 (2000).

22 Nakagomi D, Ikeda K, Okubo A et al. Ultrasound can improve the accuracy of the 2010 ACR/EULAR classification criteria for rheumatoid arthritis to predict methotrexate requirement. Arthritis Rheum. 65(4), 890-898 (2013).

23 Schmidt WA. Technology insight: the role of color and power Doppler ultrasonography in rheumatology. Nat. Clin. Pract. Rheumatol. 3(1), 35-42 (2007).

24 Balint PV, Mandl P, Kane D. 'All that glistens is not gold' - separating artefacts from true Doppler signals in rheumatological ultrasound. Ann. Rheum. Dis. 67(2), 141-142 (2008).

25 Boesen M, Ellegaard K, Boesen L et al. Ultrasound Doppler score correlates with OMERACT RAMRIS bone marrow oedema and synovitis score in the wrist joint of patients with rheumatoid arthritis. Ultraschall Med. 33(7), E166-E172 (2012).

26 Ostor AJ, Conaghan PG. Tight control in rheumatoid arthritis improves outcomes. Practitioner 253(1716), 29-32 (2009).

27 Saleem B, Brown AK, Quinn M et al. Can flare be predicted in DMARD treated RA patients in remission, and is it important? A cohort study. Ann. Rheum. Dis. 71(8), 1316-1321 (2012).

28 Luukkainen R, Ekman P, Luukkainen P, Koski JM. Ultrasonographic findings in metatarsophalangeal and talocrural joints in healthy persons. Clin. Rheumatol. 28(3), 311-313 (2009).

29 Otter SJ, Lucas K, Springett K et al. Foot pain in rheumatoid arthritis prevalence, risk factors and management: an epidemiological study. Clin. Rheumatol. 29(3), 255-271 (2010).

30 Van Der Leeden M, Steultjens MP, Ursum J et al. Prevalence and course of forefoot impairments and walking disability in the first eight years of rheumatoid arthritis. Arthritis Rheum. 59(11), 1596-1602 (2008).

31 Siddle HJ, Firth J, Waxman R, Nelson EA, Helliwell PS. A case series to describe the clinical characteristics of foot ulceration in patients with rheumatoid arthritis. Clin. Rheumatol. 31(3), 541-545 (2012).

32 Keen HI, Redmond A, Wakefield RJ et al. An ultrasonographic study of metatarsophalangeal joint pain: synovitis, structural pathology and their relationship to symptoms and function. Ann. Rheum. Dis. 70(12), 2140-2143 (2011).

33 Turner DE, Helliwell PS, Siegel KL, Woodburn J. Biomechanics of the foot in rheumatoid arthritis: identifying abnormal function and the factors associated with localised disease 'impact'. Clin. Biomech. (Bristol, Avon) 23(1), 93-100 (2008).

34 Turner DE, Helliwell PS, Emery P, Woodburn J. The impact of rheumatoid arthritis on foot function in the early stages of disease: a clinical case series. $B M C$ Musculoskelet. Disord. 7, 102 (2006).

35 Williams AE, Nester CJ, Ravey MI. Rheumatoid arthritis patients' experiences of wearing therapeutic footwear - a qualitative investigation. BMC Musculoskelet. Disord. 8 , 104 (2007)

36 Riente L, Delle Sedie A, Scire CA et al. Ultrasound imaging for the rheumatologist XXXI. Sonographic assessment of the foot in patients with rheumatoid arthritis. Clin. Exp. Rheumatol. 29(1), 1-5 (2011).

37 Scire CA, Iagnocco A, Meenagh G et al. Ultrasound imaging for the rheumatologist XXXIII. Sonographic assessment of the foot in early arthritis patients. Clin. Exp. Rheumatol. 29(3), 465-469 (2011).

38 Siddle HJ, Hodgson RJ, Redmond AC et al. MRI identifies plantar plate pathology in the forefoot of patients with rheumatoid arthritis. Clin. Rheumatol. 31(4), 621-629 (2012).

39 Bowen CJ, Edwards CJ, Hooper L et al. Improvement in symptoms and signs in the forefoot of patients with rheumatoid arthritis treated with anti-TNF therapy. J. Foot Ankle Res. 3, 10 (2010).

- Provides evidence for the inclusion of US assessment of foot joints in monitoring the 
efficacy of biological therapies for rheumatoid arthritis (RA).

40 Bowen CJ, Culliford D, Allen R et al. Forefoot pathology in rheumatoid arthritis identified with ultrasound may not localise to areas of highest pressure: cohort observations at baseline and twelve months. J. Foot Ankle Res. 4(1), 25 (2011).

41 Hooper L, Bowen CJ, Gates L et al. Prognostic indicators of foot-related disability in patients with rheumatoid arthritis: results of a prospective three-year study. Arthritis Care Res. (Hoboken) 64(8), 1116-1124 (2012).

"II Discusses the use and value of US in the assesment of RA foot disease over time.

42 Brown AK, Conaghan PG, Karim Z et al. An explanation for the apparent dissociation between clinical remission and continued structural deterioration in rheumatoid arthritis. Arthritis Rheum. 58(10), 2958-2967 (2008).

43 Wakefield RJ, Gibbon WW, Emery P. The current status of ultrasonography in rheumatology. Rheumatology (Oxford) 38(3), 195-198 (1999).

44 Bowen CJ, Culliford D, Dewbury K et al. The clinical importance of ultrasound detectable forefoot bursae in rheumatoid arthritis. Rheumatology (Oxford) 49(1), 191-192 (2010).

45 Matsos M, Harish S, Zia P et al. Ultrasound of the hands and feet for rheumatological disorders: influence on clinical diagnostic confidence and patient management. Skeletal Radiol. 38(11), 1049-1054 (2009).

46 Millot F, Clavel G, Etchepare F et al. Musculoskeletal ultrasonography in healthy subjects and ultrasound criteria for early arthritis (the ESPOIR cohort). J. Rheumatol. 38(4), 613-620 (2011).

47 Bowen CJ, Hooper L, Culliford D et al. Assessment of the natural history of forefoot bursae using ultrasonography in patients with rheumatoid arthritis: a twelve-month investigation. Arthritis Care Res. (Hoboken) 62(12), 1756-1762 (2010).

48 Gregg J, Marks P. Metatarsalgia: an ultrasound perspective. Australas. Radiol. 51(6), 493-499 (2007).

49 Gregg JM, Schneider T, Marks P. MR imaging and ultrasound of metatarsalgia - the lesser metatarsals. Radiol. Clin. North Am. 46(6), 1061-1078, vi-vii (2008).

50 Banal F, Etchepare F, Rouhier B et al. Ultrasound ability in early diagnosis of stress fracture of metatarsal bone. Ann. Rheum. Dis. 65(7), 977-978 (2006).

51 Hernandez-Diaz C, Saavedra MA, Navarro-Zarza JE et al. Clinical anatomy of the ankle and foot. Reumatol. Clin.

8(Suppl. 2), 46-52 (2012).

52 Fessell DP, Jacobson JA. Ultrasound of the hindfoot and midfoot. Radiol. Clin. North Am. 46(6), 1027-1043, vi (2008).

53 Naredo E, D'agostino MA, Wakefield RJ et al. Reliability of a consensus-based ultrasound score for tenosynovitis in rheumatoid arthritis. Ann. Rheum. Dis. 72(8), 1328-1334 (2013).

54 Bruyn GA, Moller I, Garrido J et al. Reliability testing of tendon disease using two different scanning methods in patients with rheumatoid arthritis. Rheumatology (Oxford) 51(9), 1655-1661 (2012).

55 Barn R, Turner DE, Rafferty D, Sturrock RD, Woodburn J. Tibialis posterior tenosynovitis and associated pes plano valgus in rheumatoid arthritis: EMG, multi-segment foot kinematics and ultrasound features. Arthritis Care Res. (Hoboken) 65 (4), 495-502 (2012).

" Utilizes a novel combination of US and technical functional assessment of RA foot disease.

56 Hammer HB, Kvien TK. Ultrasonography shows significant improvement in wrist and ankle tenosynovitis in rheumatoid arthritis patients treated with adalimumab. Scand. J. Rheumatol. 40(3), 178-182 (2011).

57 Suzuki T, Tohda E, Ishihara K. Power Doppler ultrasonography of symptomatic rheumatoid arthritis ankles revealed a positive association between tenosynovitis and rheumatoid factor. Mod. Rheumatol. 19(3), 235-244 (2009).

58 Baan H, Drossaers-Bakkers WK, Dubbeldam R, Buurke JJ, Nene A, van de Laar MA. Flexor Hallucis Longus tendon rupture in RA-patients is associated with MTP 1 damage and pes planus. BMC Musculoskelet. Disord. 8, 110 (2007).

59 Wakefield RJ, Balint PV, Szkudlarek M et al. Musculoskeletal ultrasound including definitions for ultrasonographic pathology. J. Rheumatol. 32(12), 2485-2487 (2005).

60 Bianchi S, Martinoli C, Gaignot C, de Gautard R, Meyer JM. Ultrasound of the ankle: anatomy of the tendons, bursae, and ligaments. Semin. Musculoskelet. Radiol. 9(3), 243-259 (2005).

61 Zayat AS, Freeston JE, Conaghan PG, Hensor EM, Emery P, Wakefield RJ. Does joint position affect US findings in inflammatory arthritis? Rheumatology (Oxford) 51(5), 921-925 (2012).

62 Bowen CJ, Dewbury K, Sampson M et al. Musculoskeletal ultrasound imaging of the plantar forefoot in patients with rheumatoid arthritis: inter-observer agreement between a podiatrist and a radiologist. J. Foot Ankle Res. 1(1), 5 (2008).
63 Jousse-Joulin S, d'Agostino MA, Marhadour $\mathrm{T}$ et al. Reproducibility of joint swelling assessment by sonography in patients with long-lasting rheumatoid arthritis (SEA-Repro study part II). J. Rheumatol. 37(5), 938-945 (2010).

64 Giacomozzi C, Martelli F, Nagel A, Schmiegel A, Rosenbaum D. Cluster analysis to classify gait alterations in rheumatoid arthritis using peak pressure curves. Gait Posture 29(2), 220-224 (2009).

65 Garrow AP, Papageorgiou AC, Silman AJ, Thomas E, Jayson MI, Macfarlane GJ. Development and validation of a questionnaire to assess disabling foot pain. Pain 85(1-2), 107-113 (2000).

66 Helliwell P, Reay N, Gilworth G et al. Development of a foot impact scale for rheumatoid arthritis. Arthritis Rheum. 53(3), 418-422 (2005).

67 Vidmar G, Novak P. Reliability of in-shoe plantar pressure measurements in rheumatoid arthritis patients. Int. J. Rehabil. Res. 32(1), 36-40 (2009).

68 Turner DE, Woodburn J. Characterising the clinical and biomechanical features of severely deformed feet in rheumatoid arthritis. Gait Posture 28(4), 574-580 (2008).

69 Khazzam M, Long JT, Marks RM, Harris GF. Kinematic changes of the foot and ankle in patients with systemic rheumatoid arthritis and forefoot deformity. J. Orthop. Res. 25(3), 319-329 (2007).

70 Siddle HJ, Hodgson RJ, O'connor P et al. Magnetic resonance arthrography of lesser metatarsophalangeal joints in patients with rheumatoid arthritis: relationship to clinical, biomechanical, and radiographic variables. J. Rheumatol. 39(9), 1786-1791 (2012).

71 Woodburn J, Hennessy K, Steultjens MP, Mcinnes IB, Turner DE. Looking through the 'window of opportunity': is there a new paradigm of podiatry care on the horizon in early rheumatoid arthritis? J. Foot Ankle Res. 3, 8 (2010).

72 Turner DE, Helliwell PS, Woodburn J. Methodological considerations for a randomised controlled trial of podiatry care in rheumatoid arthritis: lessons from an exploratory trial. BMC Musculoskelet. Disord. 8, 109 (2007).

73 Williams AE, Davies S, Graham A et al. Guidelines for the Management of the Foot Health Problems Associated with Rheumatoid Arthritis. Musculoskeletal Care 9(2), 86-92 (2011).

74 Symmons DP. Rheumatoid arthritis: assessing disease activity and outcome. Clin. Med. 10(3), 248-251 (2010). 
75 Lloyd S, Bujkiewicz S, Wailoo AJ, Sutton AJ, Scott D. The effectiveness of anti-TNF-alpha therapies when used sequentially in rheumatoid arthritis patients: a systematic review and meta-analysis. Rheumatology (Oxford) 49(12), 2313-2321 (2010).

76 Herenius MM, Hoving JL, Sluiter JK et al. Improvement of work ability, quality of life, and fatigue in patients with rheumatoid arthritis treated with adalimumab. J. Occup. Environ. Med. 52(6), 618-621 (2010).

77 Combe B, Codreanu C, Fiocco U et al. Efficacy, safety and patient-reported outcomes of combination etanercept and sulfasalazine versus etanercept alone in patients with rheumatoid arthritis: a double-blind randomised 2-year study. Ann. Rheum. Dis. 68(7), 1146-1152 (2009).

78 van der Leeden M, Steultjens MP, Van Schaardenburg D, Dekker J. Forefoot disease activity in rheumatoid arthritis patients in remission: results of a cohort study. Arthritis Res. Ther. 12(1), R3 (2010).

79 Prevoo ML, van 't Hof MA, Kuper HH, van Leeuwen MA, van de Putte LB, van Riel PL. Modified disease activity scores that include twenty-eight-joint counts. Development and validation in a prospective longitudinal study of patients with rheumatoid arthritis. Arthritis Rheum. 38(1), 44-48 (1995).

80 D'agostino MA, Ayral X, Baron G, Ravaud P, Breban M, Dougados M. Impact of ultrasound imaging on local corticosteroid injections of symptomatic ankle, hind-, and mid-foot in chronic inflammatory diseases. Arthritis Rheum. 53(2), 284-292 (2005).

81 Drakonaki EE, Kho JS, Sharp RJ, Ostlere SJ. Efficacy of ultrasound-guided steroid injections for pain management of midfoot joint degenerative disease. Skeletal Radiol. 40(8), 1001-1006 (2011).

82 Dougados M, Jousse-Joulin S, Mistretta F et al. Evaluation of several ultrasonography scoring systems for synovitis and comparison to clinical examination: results from a prospective multicentre study of rheumatoid arthritis. Ann. Rheum. Dis. 69(5), 828-833 (2010).

83 Marhadour T, Jousse-Joulin S, Chales G et al. Reproducibility of joint swelling assessments in long-lasting rheumatoid arthritis: influence on Disease Activity Score-28 values (SEA-Repro study part I). J. Rheumatol. 37(5), 932-937 (2010).

84 Naredo E, Bijlsma JW, Conaghan PG et al. Recommendations for the content and conduct of European League Against Rheumatism (EULAR) musculoskeletal ultrasound courses. Ann. Rheum. Dis. 67(7), 1017-1022 (2008).

85 Mcalindon T, Kissin E, Nazarian L et al. American College of Rheumatology report on reasonable use of musculoskeletal ultrasonography in rheumatology clinical practice. Arthritis Care Res. (Hoboken) 64(11), 1625-1640 (2012).

86 Brown AK, Roberts TE, O'connor PJ, Wakefield RJ, Karim Z, Emery P.

The development of an evidence-based educational framework to facilitate the training of competent rheumatologist ultrasonographers. Rheumatology (Oxford) 46(3), 391-397 (2007).

- Introduces the concept of tailored learning for US in discrete areas of practice, such as RA foot disease.

87 D'agostino MA, Conaghan PG, Naredo E et al. The OMERACT Ultrasound Task Force - advances and priorities. J. Rheumatol. 36(8), 1829-1832 (2009).

88 Hammer HB, Kvien TK. Comparisons of 7- to 78-joint ultrasonography scores: all different joint combinations show equal response to adalimumab treatment in patients with rheumatoid arthritis. Arthritis Res. Ther. 13(3), R78 (2011).

89 Scire CA, Montecucco C, Codullo V, Epis O, Todoerti M, Caporali R. Ultrasonographic evaluation of joint involvement in early rheumatoid arthritis in clinical remission: power Doppler signal predicts short-term relapse. Rheumatology (Oxford) 48(9), 1092-1097 (2009).

90 Filer A, De Pablo P, Allen G et al. Utility of ultrasound joint counts in the prediction of rheumatoid arthritis in patients with very early synovitis. Ann. Rheum. Dis. 70(3), 500-507 (2011).

91 Hammer HB, Sveinsson M, Kongtorp AK, Kvien TK. A 78-joints ultrasonographic assessment is associated with clinical assessments and is highly responsive to improvement in a longitudinal study of patients with rheumatoid arthritis starting adalimumab treatment. Ann. Rheum. Dis. 69(7), 1349-1351 (2010).

92 Naredo E, Gamero F, Bonilla G, Uson J, Carmona L, Laffon A. Ultrasonographic assessment of inflammatory activity in rheumatoid arthritis: comparison of extended versus reduced joint evaluation. Clin. Exp. Rheumatol. 23(6), 881-884 (2005).

93 Naredo E, Valor L, De la Torre I et al. Ultrasound joint inflammation in rheumatoid arthritis in clinical remission: how many and which joints should be assessed? Arthritis Care Res. (Hoboken) 65(4), 512-517 (2013).
- Discusses the merits of assessment of RA disease by reduced joint scores and includes discussion of foot joint counts.

94 Backhaus M, Ohrndorf S, Kellner $\mathrm{H}$ et al. Evaluation of a novel 7-joint ultrasound score in daily rheumatologic practice: a pilot project. Arthritis Rheum. 61(9), 1194-1201 (2009).

95 Perricone C, Ceccarelli F, Modesti M et al. The 6-joint ultrasonographic assessment: a valid, sensitive-to-change and feasible method for evaluating joint inflammation in RA. Rheumatology (Oxford) 51(5), 866-873 (2012).

96 Naredo E, Rodriguez M, Campos C et al. Validity, reproducibility, and responsiveness of a twelve-joint simplified power Doppler ultrasonographic assessment of joint inflammation in rheumatoid arthritis. Arthritis Rheum. 59(4), 515-522 (2008).

97 Klauser AS. Value of contrast-enhanced ultrasound in rheumatic disease. Eur. Radiol. 15(Suppl. 5), E117-E122 (2005).

98 Naredo E, Moller I, Acebes C et al. Three-dimensional volumetric ultrasonography. Does it improve reliabililty of musculoskeletal ultrasound? Clin. Exp. Rheumatol. 28(1), 79-82 (2010).

99 Iagnocco A, Perella C, D’agostino MA, Sabatini E, Valesini G, Conaghan PG. Magnetic resonance and ultrasonography real-time fusion imaging of the hand and wrist in osteoarthritis and rheumatoid arthritis. Rheumatology (Oxford) 50(8), 1409-1413 (2011).

\section{Websites}

101 Arthritis Research UK video resources. Musculoskeletal ultrasound: a beginner's guide to normal peripheral joint anatomy. www.arthritisresearchuk.org/healthprofessionals-and-students/video-resources/ msus/foot-scans.aspx (Accessed 17 May 2013)

102 Grassi W. Advanced rheumatology sonography. Università Politecnicadelle Marche, Rome, Italy. www.e-sonography.com/rheuma (Accessed 17 May 2013)

103 Geertsma T. Ultrasound Cases. Musculoskeletal Joints and Tendons, 6.8 Foot. Hospital GelderseVallei in Ede, The Netherlands and ALOKA Holding Europe AG in Zug, Switzerland. www.ultrasoundcases.info/category. aspx?cat $=112$

(Accessed 17 May 2013) 
Reproduced with permission of the copyright owner. Further reproduction prohibited without permission. 